Ann. Génét. Sél. anim., I969, 1 (2), II9-I30.

\title{
ECTROMÉLIE BITHORACIQUE HÉRÉDITAIRE CHEZ LE CHIEN
}

\author{
J. LADRAT, P.-C. BLIN, et J.-J. LAUVERGNE \\ Laboratoire de Zootechnie, École nationale supérieure agronomique, 78-Grignon; \\ Laboratoire d'Anatomie comparée, École nationale vétérinaire, 94-Alfort; \\ Station centrale de Génétique animale, \\ Centre national de Recherches zootechniques, 78-Jouy-en-Josas \\ Institut national de la Recherche agronomique
}

\section{SOMMAIRE}

Des cas d'ectromélie bithoracique ont été observés chez des animaux des deux sexes dans trois générations de chiens. L'anomalie est simple et sa manifestation est à peu près constante. Les animaux porteurs sont viables mais, éprouvant des difficultés à se déplacer dans le jeune âge, ils sont d'un élevage plus difficile. Les données génétiques recueillies indiquent que l'anomalie est sous la dépendance d'un gène autosomal récessif.

Il y a quelques années, l'un d'entre nous eut l'occasion d'acquérir une chienne ectromèle bithoracique. L'animal, du nom de Yama, était né en I950, dans une ferme de l'Ariège. Sans être inscrite au livre des origines, elle appartenait à la race de Chien courant de l'Ariège : petite taille, oreilles tombantes, pelage noir et feu, avec une assez grande extension de la panachure blanche. Amenée à l'École nationale d'Agriculture de Grignon, elle servit à des expériences de croisements ( $\left.{ }^{1}\right)$.

\section{I. - ÉTUDE DESCRIPTIVE}

\section{A. - La mère}

Yama vécut 5 ans; c'est sur elle que nous avons les renseignements les plus complets.

Extérieurement, 1a seule anomalie résidait dans l'absence totale de la partie libre des membres thoraciques; on distinguait toutefois, de chaque côté, sous la

(1) VERGER (1967) a fait état, dans sa thèse, de quelques-uns des résultats présentés ici et I,ADRAT (1967) en a donné une brève relation. 
peau, des reliefs osseux qui ne pouvaient être qu'une scapula et qu'une portion de l'humérus engagée dans la cavité glénoïde. Au repos, l'animal se tenait en décubitus sternal. Pour se déplacer, il se mettait debout en se dressant sur ses membres postérieurs (fig. I); il marchait en plantigrade, contrairement aux chiens de cirque qui, dans la même position, demeurent digitigrades; ses épaules oscillaient en synchronisme avec le déplacement des membres pelviens.

La station debout semblait conférer à l'animal une certaine prééminence sur ses congénères quadrupèdes : ainsi, lorsque la chienne, agacée par ses camarades, s'élevait sur ses membres postérieurs, toute agacerie cessait et leurs auteurs s'éloignaient. HEDIGER et SiRCOM (I955) ont fait les mêmes observations sur des carnivores maintenus en captivité.

L'anomalie n'empêcha pas Yama d'avoir quatre portées; ce fut même une bonne nourrice. Lors des accouplements, on dut cependant soutenir la chienne par le poitrail.

Anatomiquement, les anomalies relevées ne portaient que sur les membres thoraciques.

De chaque côté, seules la scapula et une petite partie de l'humérus sont représentées (fig. 2). L'angle ventral de la scapula (angulus ventralis) est étiré et la cavité glénoïde (cavitas glenoidalis) est plus ouverte que normalement; l'angle caudal (angulus caudalis) s'incurve fortement en arrière.

Les divers reliefs de l'os, épine scapulaire (spina scapulae), pointe acromiale (processus hamatus), paracromion (processus suprahamatus), apophyse coracoïde (processus coracoideus) sont peu marqués, effacés.

La pièce humérale, irrégulièrement parallélépipédique, s'oppose imparfaitement à l'angle ventral de la scapula; elle s'incurve et déborde cet angle en avant.

Le muscle brachio-céphalique ( $m$. brachiocephalicus), les muscles de l'épaule, le grand dorsal ( $m$. latissimus dorsi), dont l'insertion inférieure se fait normalement en divers points de l'humérus, concentrent leurs attaches au pourtour de la "pièce humérale " et constituent, avec les pectoraux amincis et confondus en une "lame pectorale ", un " revêtement musculaire enveloppant " qui recouvre le moignon osseux en question. Le biceps brachial ( $m$. biceps brachii), le sous-scapulaire ( $m$. subscapularis) et le grand rond ( $m$. teres major) sont peu développés; le triceps brachial (triceps brachii), également réduit, se fixe en arrière du moignon huméral.

L'anomalie décrite chez Yama n'est pas sans rappeler celle que nous avions observée précédemment au bipède antérieur d'une chèvre (BLIN et OVEJERO DEL, AGUA, I952). Ajoutons que, sur le plan vasculaire, l'artère sous-clavière ( $a$. subclavia) se divise, à sa terminaison, en deux fortes branches dont le territoire de drainage rappelle assez bien celui d'une artère sus-scapulaire (a. suprascapularis) et d'une artère sous-scapulaire (a. subscapularis). L'artère axillaire (a. axillaris) prolongement de l'artère sous-clavière (MILIER et al., I964) n'est donc pas représentée. Le plexus brachial (plexus brachialis) est peu développé; outre ses branches $\mathrm{du}$ tronc, il fournit des rameaux ténus aux seuls muscles du membre thoracique représentés. Corollaire, le bulbe brachial de la moelle épinière (intumescentia cervicalis) est peu marqué. 


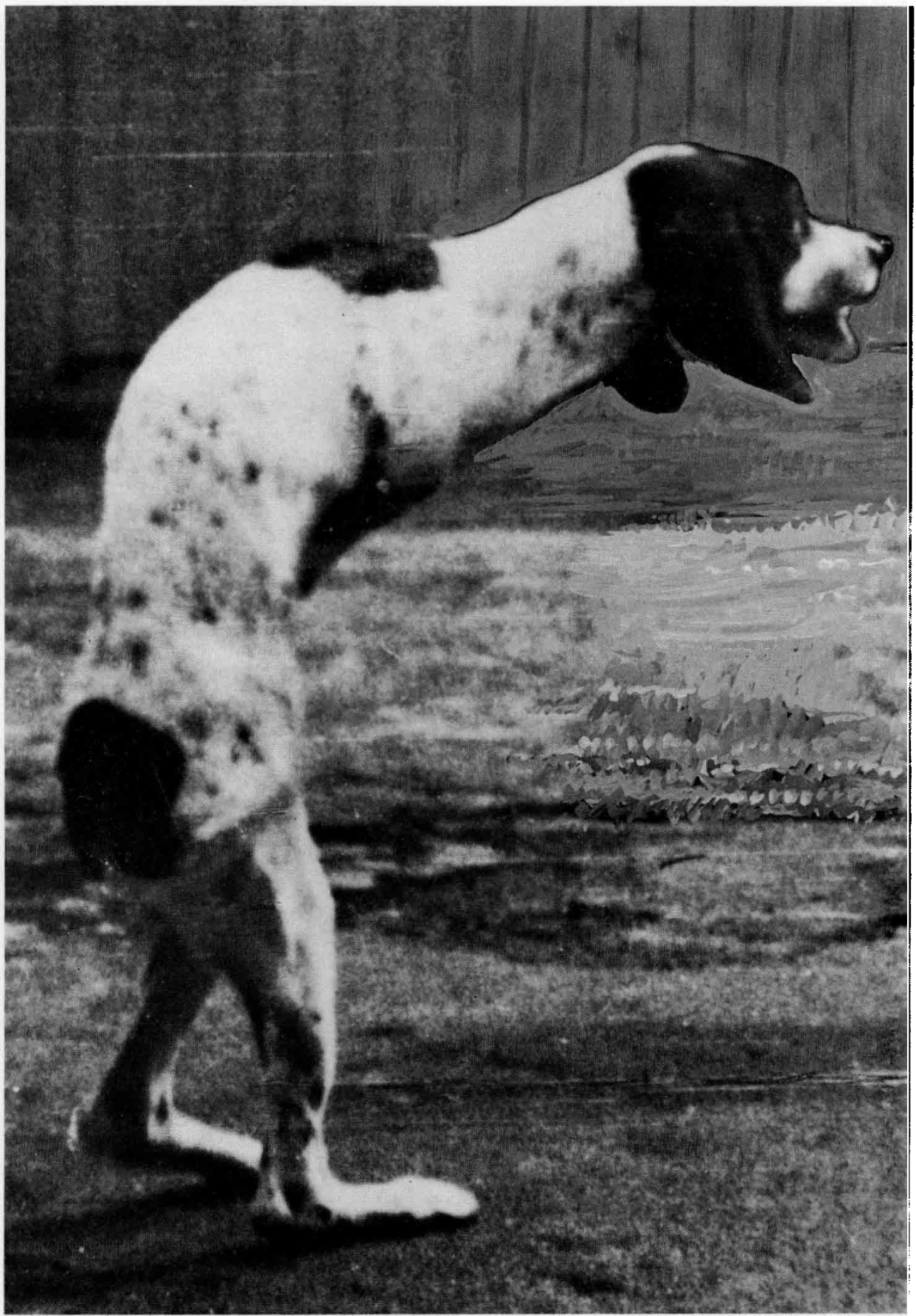

FIG. I. - La démarche caractéristique de la mère, YAMA, érigée en position plantigrade. 


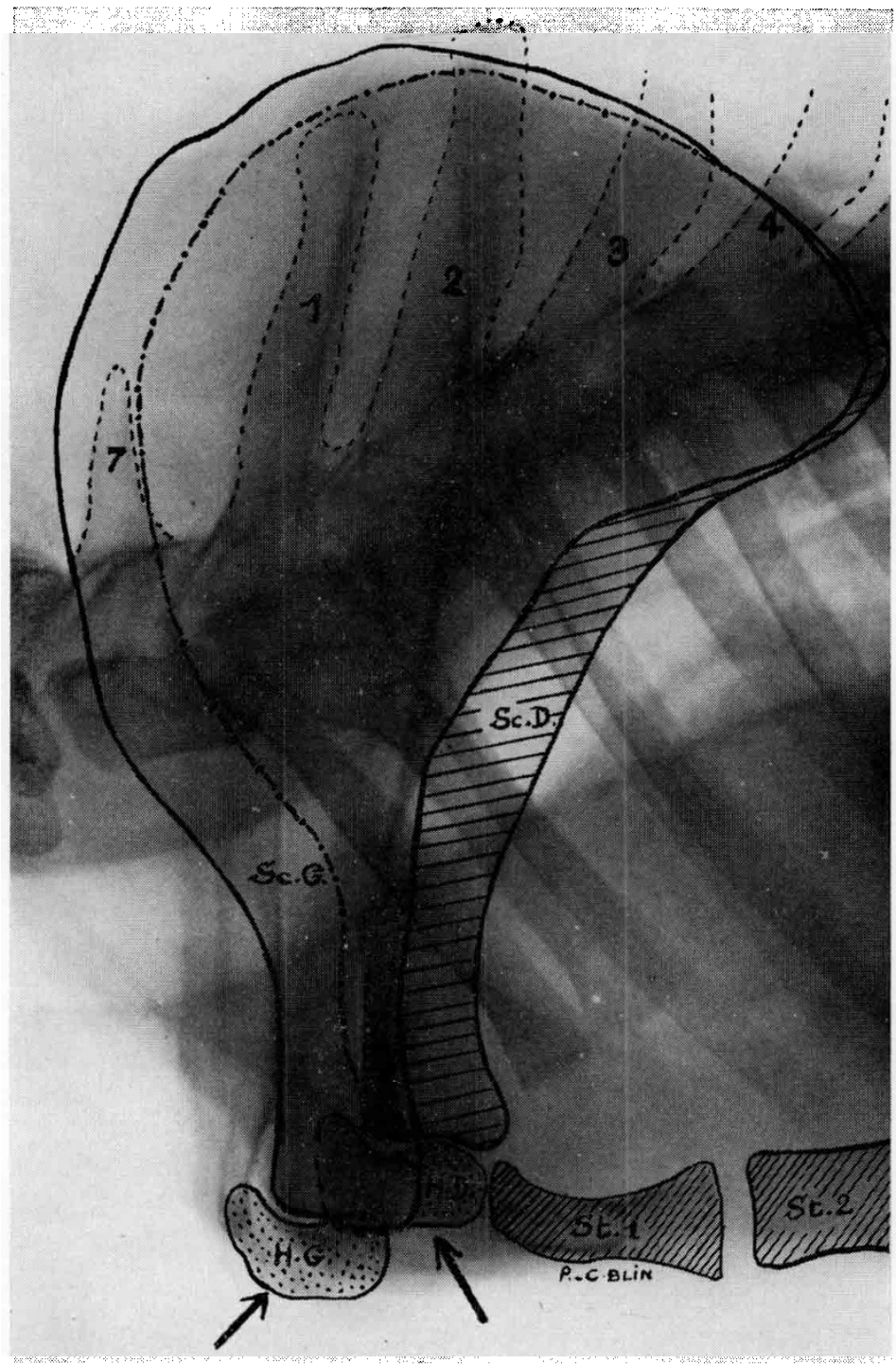

FIG. 2. - Le squelette des membres thoraciques de YAMA, chienne ectromèle.

I,es scapulae sont déformées, légèrement décalées l'une par rapport à l'autre; leur angle caudal est fortement incurvé en arrière.

Le col de la scapula droite, au lieu d'être bref et large comme c'est le cas normalement chez le chien, est long, rétréci. Ia cavité glénoïde de chaque scapula est largement ouverte.

L,es " pièces humérales " sont aplaties dorso-ventralement, relevées en avant et en haut.

\section{Légendes :}

- I à $4:$ apophyse épineuse des 4 premières vertèbres thoraciques;

- 7 : apophyse épineuse de la $7^{\mathbf{e}}$ vertèbre cervicale;

- H. G. : " pièce humérale "gauche;

- H. D.: " pièce humérale "droite;

- Sc. G. : scapula gauche;

- Sc. D. : scapula droite. 


\section{B. - Les descendants}

Sur 36 descendants de première et deuxième génération, la chienne Yama eut I7 ectromèles (II mâles, 6 femelles); la durée de gestation fut normale (6o à 63 jours). Deux chiots étaient mort-nés, I4 moururent dans la première semaine, un seul sujet, un mâle, put être élevé jusqu'à 1'âge de I5 mois. Tous les animaux étaient de taille normale. Cette forte mortalité post-natale semble due, en partie, aux difficultés d'élevage et d'entretien des chiots anormaux; ceux-ci, handicapés par leur infirmité, éprouvaient les plus grandes difficultés pour téter. Le seul animal ectromèle qui vécut, prit spontanément, vers l'âge de 6 semaines, les mêmes attitudes statiques et dynamiques que sa grand-mère.

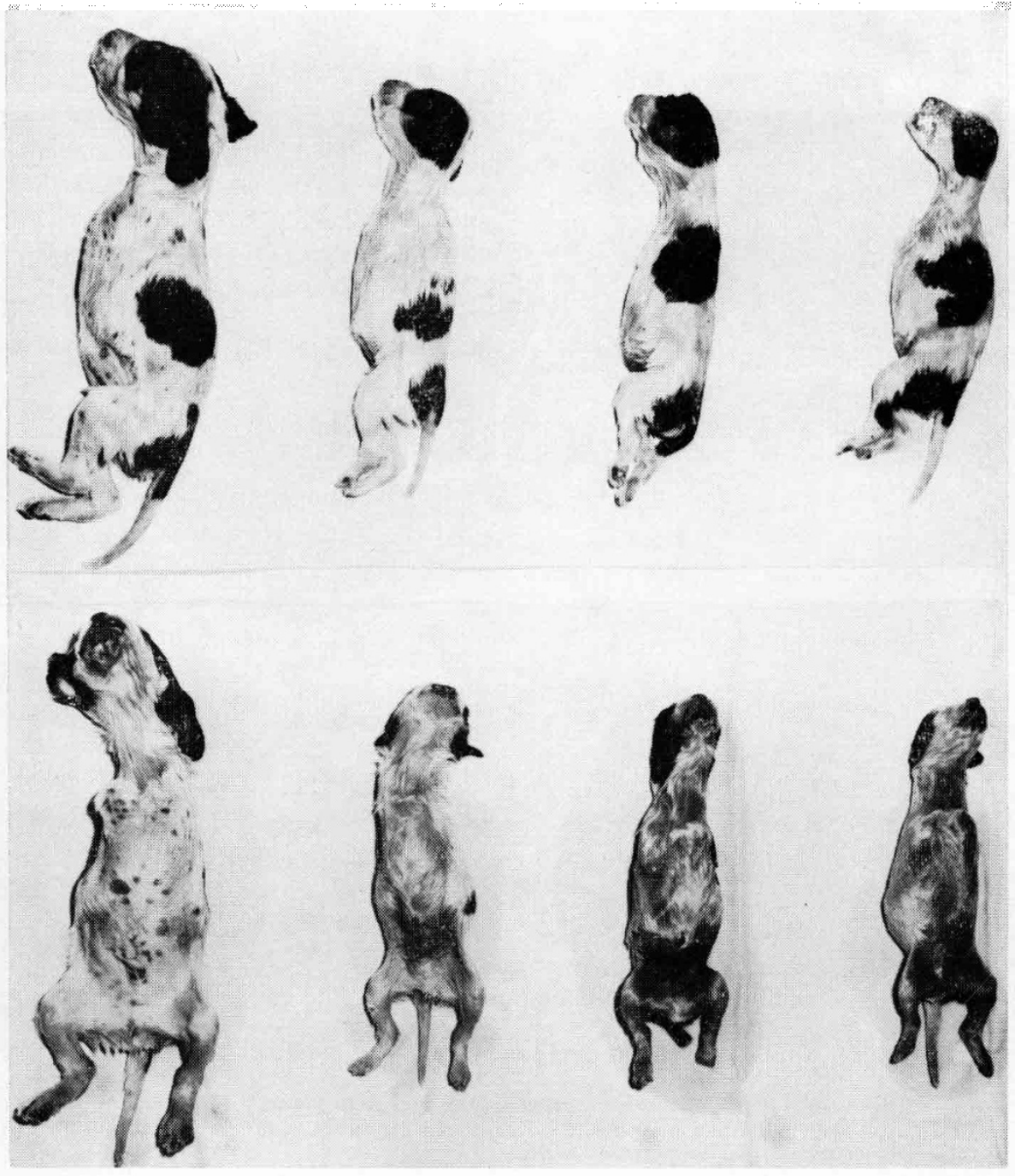

FIG. 3. - Ectromélie familiale chez le chien: quèques descenàants ảe Y $\mathrm{Y} M A$ (cliché Montabrun). 

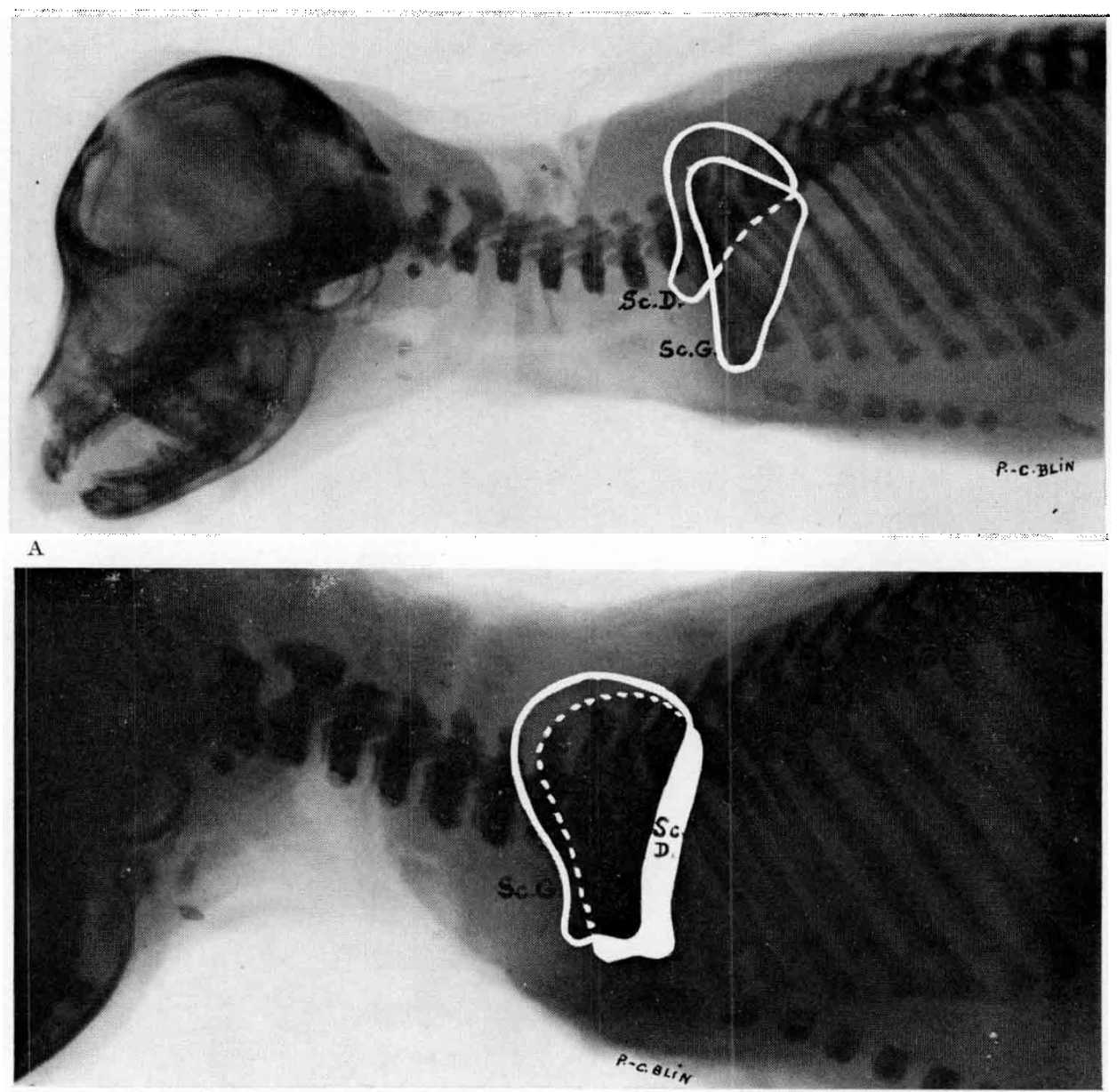

B

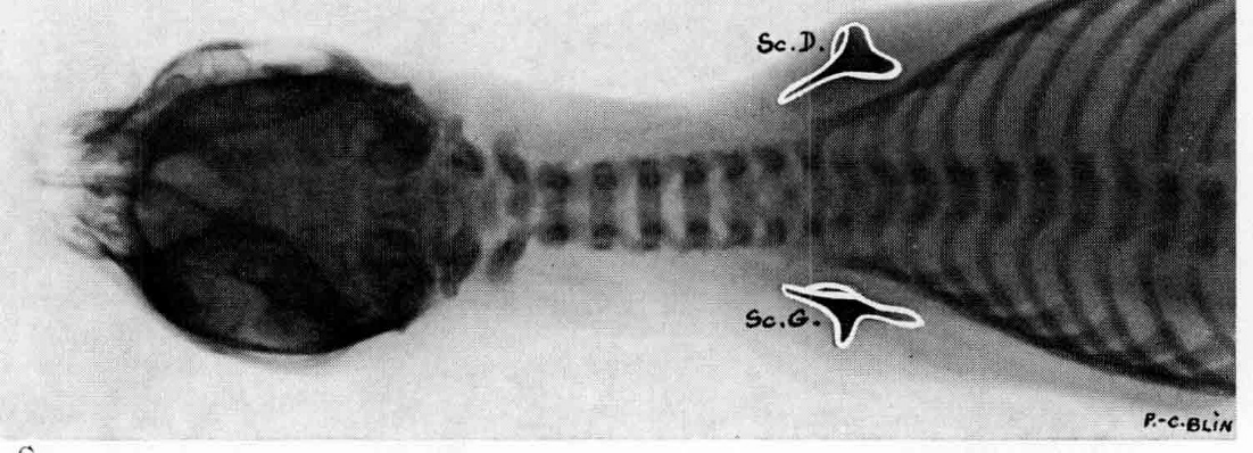

c

Frg. 4. - Clichés radiographiques du squelette thoracique de trois petits-fils de YAMA, nés le 26 sept. 1954. Les scapulae, dont le contour a été figuré en blanc sont déformées et situées asymétriquement (A et B) ou symétriquement (C) par rapport au thorax.

Légendes :

- Sc. D : scapula droite;

- Sc. G. : scapula gauche. 
Extérieurement, les animaux ressemblaient fort à leur mère ou grand-mère. Un des chiots, une femelle, présentait, en outre, un bec-de-lièvre.

Les anomalies observées chez les chiots rappellent celles décrites chez Yama; elles affectent une remarquable symétrie et diffèrent peu d'un sujet à l'autre.

Le squelette du membre thoracique ne comporte qu'une seule pièce, la scapula (fig. 4); en raison de 1'absence de "pièce humérale", les muscles qui, chez la mère, se fixaient sur cette dernière, s'attachent au pourtour de l'angle ventral de la scapula en formant souvent une sorte d'aponévrose résistante qui enveloppe le moignon glénoïdien. Un type de musculature de chiot ectromèle est représenté figure 5 .

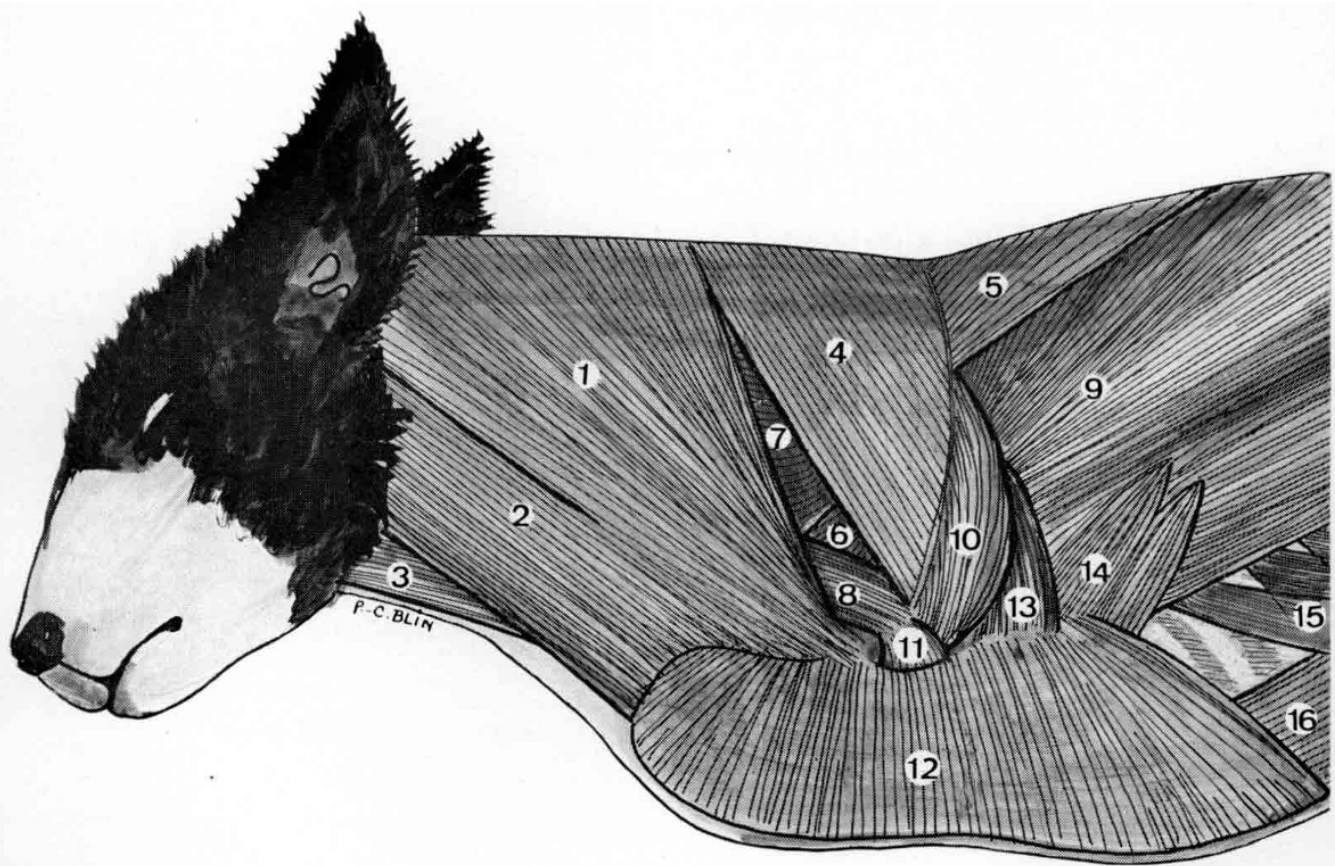

FIG. 5. - Muscles superficiels $d u$ cou, de l'épaule et du thorax d'un chiot ectromèle, fils de YAMA.

I - M. cléido-cervical ( $m$. cleidocervicalis).

2 - M. sterno-céphalique ( $m$. sternocephalicus).

3 - M. sterno-hyoïdien ( $m$. sternohyoideus).

4 - M. trapèze, portion cervicale (m. trapezius, pars cervicalis).

5 - M. trapeze, portion thoracique (m. trapezius, pars thoracica).

6 - M. sus-épineux ( $m$. supraspinatus).

7 - M. dentelé ventral du cou, angulaire de l'épaule ( $m$. serratus ventralis cervicis).

8 - M. omo-transversaire ( $m$. omotransversarius).

9 - M. large du dos, grand dorsal (m. latissimus dorsi).

ro - M. deltoìde, portion scapulaire ( $m$. deltoideus, pars scapularis).

II - M. deltoïde, portion acromienne $(m$. deltoideus, pars acromialis).

r2 - Ms. pectoraux ( $M m$. pectorales).

I3 - M. triceps brachial (m. triceps brachii).

r - Lame musculaire non définie dont les fibres se perdent sur le large du dos et sous les pectoraux.

I 5 - M. oblique externe de l'abdomen, premières dentelures costales ( $m$. obliquus externus abdominis).

I6 - M. droit de l'abdomen ( $m$. rectus abdominis).

Remarque: Aux variations morphologiques de détail près, chez tous les chiots ectromèles nés de YAMA, dépourvus d'humérus, les muscles du cou, de l'épaule et du thorax ayant normalement une insertion inférieure humérale s'unissent sous des formes diverses pour englober l'angle ventral (angulus ventralis) de la scapula, en s'y fixant partiellement. 
Comme chez Yama, les branches du plexus brachial sont particulièrement ténues et ceci bilatéralement. Le bulbe brachial, ainsi que les racines dorsale et ventrale des nerfs rachidiens qui rentrent dans la constitution du plexus brachial, sont peu développés, comparativement à ceux d'un chiot normal de même race et de même âge. Ces faits ont été évoqués également par SALMON (I908) dans son étude des modifications du système nerveux qui accompagnent 1'ectromélie et par SPENGLER (I950) dans sa thèse sur la péromélie du chat.

Signalons enfin que, chez les chiots ectromèles que nous avons disséqués, l'anse sous-clavière ou anse de Vieussens (ansa subclavia) ne se forme pas à l'entour de 1'artère sous-clavière. Nous l'avions déjà mentionné chez ces mêmes chiens dans notre étude comparée du sympathique thoracique (BLIN, I958).

\section{II. - ÉTUdE GÉNÉTIQUE}

La chienne Yama a été tout d'abord croisée à un mâle fox-terrier à poil ras de même format; elle a donné 4 produits, tous normaux.

Unie ultérieurement à l'un d'eux, elle mit au monde en 3 portées r2 animaux dont 7 ectromèles. Enfin, des croisements de type $\mathrm{F}_{2}$ ont été réalisés : 7 portées totalisant 23 descendants dont I3 ectromèles (tabl.r).

TABLEAU I

Détail des croisements expérimentaux réalisés à Grignon

\begin{tabular}{|c|c|c|c|c|c|c|c|c|c|c|}
\hline \multicolumn{4}{|c|}{ Croisements } & \multicolumn{7}{|c|}{ Descendants } \\
\hline \multirow{2}{*}{ Type } & \multirow{2}{*}{ Père } & \multirow{2}{*}{ Mère } & \multirow{2}{*}{$\begin{array}{l}\text { Nombre } \\
\text { de } \\
\text { portées }\end{array}$} & \multicolumn{3}{|c|}{ Normaux } & \multicolumn{3}{|c|}{ Ectromèles } & \multirow{2}{*}{$\begin{array}{c}\text { Total } \\
\text { par } \\
\text { lignes }\end{array}$} \\
\hline & & & & రేరే & 우우 & total & పై & 우우 & total & \\
\hline$F_{I}$ & $\begin{array}{c}\text { Fox } \\
\text { terrier }\end{array}$ & Yama & I & I & 3 & 4 & 0 & 0 & 0 & 4 \\
\hline $\mathrm{BC}$ & $A\left({ }^{1}\right)$ & Yama & 3 & 4 & $\mathbf{I}$ & 5 & 4 & 3 & 7 & 12 \\
\hline \multirow[t]{2}{*}{$\mathrm{F}_{2}$} & $\begin{array}{l}\mathrm{A} \\
\mathrm{A} \\
\mathrm{B}\left({ }^{3}\right)\end{array}$ & $\begin{array}{ll}a & \left({ }^{2}\right) \\
b & (2) \\
a & \end{array}$ & $\begin{array}{l}3 \\
3 \\
I\end{array}$ & $\begin{array}{l}\mathbf{I} \\
\mathbf{I} \\
\mathbf{I}\end{array}$ & $\begin{array}{l}6 \\
I \\
3\end{array}$ & $\begin{array}{l}7 \\
2 \\
4\end{array}$ & $\begin{array}{l}0 \\
6 \\
4\end{array}$ & $\begin{array}{l}I \\
2 \\
0\end{array}$ & $\begin{array}{l}\text { I } \\
8 \\
\text { I }\end{array}$ & $\begin{array}{r}8 \\
10 \\
5\end{array}$ \\
\hline & Total $\mathrm{F}_{2}$ & 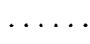 & 7 & 3 & Io & I3 & 7 & 3 & IO & 23 \\
\hline
\end{tabular}

(1) A est le fils de YAMA et du mâle fox-terrier terrier.

(2) $a$ et $b$ sont deux filles issues du même croisement que $A$.

(3) B est un mâle normal issu du back-cross $\mathrm{A} \times$ YAMA. 
Ces résultats plaident en faveur de l'hypothèse d'un déterminisme monofactoriel autosomal récessif, hypothèse qui est mise à l'épreuve dans le tableau 2.

Les résultats de première génération et du back-cross sont en bon accord avec 1'hypothèse. Ceux de la $\mathrm{F}_{2}$ semblent, à première vue, la contredire mais, avec la correction de YEATES, pour les petits effectifs, on retrouve des écarts non significatifs au seuil de 5 p. Ioo.

TABLEAU 2

Test de l'hypothèse d'un facteur récessif autosomal

\begin{tabular}{|c|c|c|c|c|c|c|c|c|}
\hline \multirow{3}{*}{$\begin{array}{l}\text { Type } \\
\text { de } \\
\text { croisement }\end{array}$} & \multicolumn{5}{|c|}{ Nombre de descendants } & \multirow{3}{*}{$\chi^{2}$} & \multirow{3}{*}{$v$} & \multirow{3}{*}{$\begin{array}{l}\text { Probabilité } \\
\text { que la valeur de } \chi^{2} \\
\text { soit dépassée }\end{array}$} \\
\hline & \multicolumn{2}{|c|}{ Normaux } & \multicolumn{2}{|c|}{ Ectromèles } & \multirow{2}{*}{ Total } & & & \\
\hline & observés & calculés & observés & calculés & & & & \\
\hline$F_{\mathbf{I}}$ & 4 & 4 & o & 0 & 4 & & & \\
\hline Back-Cross & 5 & 6 & 7 & 6 & 12 & 0,33 & $\mathbf{I}$ & 0, Io $<\mathrm{P}$ \\
\hline$F_{2}$ & I3 & I 7,25 & Io & 5,75 & 23 & $\begin{array}{l}4,188 \\
3,260\left({ }^{1}\right)\end{array}$ & $\begin{array}{l}\text { I } \\
\text { I }\end{array}$ & $\begin{array}{c}0,025<\mathrm{P}<0,05 \\
0,05<\mathrm{P}\end{array}$ \\
\hline
\end{tabular}

(1) Avec la correction de YEATES.

\section{III. - DISCUSSION ET CONCLUSION}

Bien que Burns et Fraser (1966) n'en fassent pas état dans leur traité de génétique du Chien, plusieurs cas d'ectromélie bithoracique à allure héréditaire sont rapportés par différents auteurs. Nous avons essayé de réunir dans le tableau 3 les renseignements, très souvent incomplets, qui les concernent.

Les descriptions coïncident assez bien avec celles que nous avons données plus haut : la démarche est plantigrade (cas 5, 8, 9), avec des mouvements synchrones des scapulae (cas 8); ces os sont les seuls représentés aux membres thoraciques (cas 5); le bec-de-lièvre est quelquefois observé en association avec l'ectromélie.

Quant à la transmission héréditaire, elle semble indéniable dans plusieurs cas $(\mathrm{I}, 2,3,6)$, probable dans les cas 4 et 7 . Les cas $I, 2$ et 6 peuvent s'expliquer par un gène autosomal récessif; cette récessivité serait alors confirmée par les observations 4 et 7 . Sur le cas $n^{0} 3$, il est difficile de conclure. 


\section{TABLEAU 3}

Cas d'ectromélie bithoracique à allure héréditaire velevés dans la littérature

\begin{tabular}{|c|c|c|c|}
\hline $\begin{array}{c}\text { Cas } \\
n^{\circ}\end{array}$ & $\begin{array}{l}\text { Nom des observateurs } \\
\text { et expérimentateurs, } \\
\text { date et lieu }\end{array}$ & $\begin{array}{l}\text { Renseignements descriptifs } \\
\text { et génétiques }\end{array}$ & Auteurs \\
\hline I & $\begin{array}{l}\text { AucANTE, date et lieu non } \\
\text { précisés. }\end{array}$ & $\begin{array}{l}\text { Une chienne normale }\left({ }^{1}\right) \text { a donné } 4 \text { por- } \\
\text { tées composées au total de } 7 \text { chiots } \\
\text { normaux et de I } 3 \text { chiots ectromèles- } \\
\text { bithoraciques affectés de bec-de-lièvre. }\end{array}$ & $\begin{array}{l}\text { GEOFFROY SAINT-HI- } \\
\text { LAIRE (I } 836)\end{array}$ \\
\hline 2 & $\begin{array}{l}\text { L'auteur, date et lieu non } \\
\text { précisés. }\end{array}$ & $\begin{array}{l}\text { Une chienne normale très vieille ayant } \\
\text { mis bas un grand nombre de fois a eu } \\
\text { successivement } 2 \text { portées l'une de } 4 \\
\text { chiots normaux, un ectromèle, l'au- } \\
\text { tre de } 3 \text { chiots ectromèles }\left({ }^{1}\right) \text {. }\end{array}$ & 一 \\
\hline 3 & $\begin{array}{l}\text { L'auteur, date et lieu non } \\
\text { précisés. }\end{array}$ & $\begin{array}{l}\text { Une chienne et son fils étaient tous les } \\
\text { deux ectromèles bithoraciques. La } \\
\text { chienne avait eu plusieurs autres petits } \\
\text { dont quelques-uns au moins, si ce } \\
\text { n'est tous, étaient ectromèles. }\end{array}$ & - \\
\hline 4 & $\begin{array}{l}\text { L'auteur, dans les années } \\
\text { I } 880 \text {, à l'École vétéri- } \\
\text { naire d'Alfort. }\end{array}$ & $\begin{array}{l}\text { Une chienne ectromèle bithoracique a } \\
\text { donné } 40 \text { petits, tous normaux }{ }^{\left({ }^{2}\right)}\end{array}$ & BARRIER, I 883 \\
\hline 5 & $\begin{array}{l}\text { Les auteurs, à l'École vété- } \\
\text { rinaire de Lyon ont dis- } \\
\text { séqué un animal fourni } \\
\text { par M. BERTHEOL, vété- } \\
\text { rinaire à Ambert (Puy- } \\
\text { de-Dôme), qui a fait les } \\
\text { observations sur place. }\end{array}$ & $\begin{array}{l}\text { Deux chiots ectromèles sont nés d'une } \\
\text { chienne anatomiquement normale, } \\
\text { mais atteinte de " chorée " des mem- } \\
\text { bres antérieurs. Les animaux ont une } \\
\text { démarche plantigrade et se déplacent } \\
\text { surtout par bonds. A la dissection, on } \\
\text { observe les seules scapulae plus étroi- } \\
\text { tes que normalement, convexes et dé- } \\
\text { pourvues de cavité glénoïde et d'apo- } \\
\text { physe coracoïde. La forte arcure des } \\
\text { premières côtes donne à la poitrine } \\
\text { une forme moins comprimée que d'ha- } \\
\text { bitude. }\end{array}$ & $\begin{array}{l}\text { LESBRE et FORGEOT } \\
\text { (I9O2) }\end{array}$ \\
\hline 6 & $\begin{array}{l}\text { L'auteur, à partir de roo6 } \\
\text { à Pise }\end{array}$ & $\begin{array}{l}\text { Une femelle de race chien-loup italien } \\
\text { couverte par un mâle de même race a } \\
\text { engendré } 3 \text { portées parmi lesquelles } \\
\text { on observait des chiots ectromèles. Le } \\
\text { même mâle a également donné des ec- } \\
\text { tromèles, en croisement avec une fe- } \\
\text { melle terrier. }\end{array}$ & LISI (I909). \\
\hline
\end{tabular}

(1) Accouplée sans doute à des mâles normaux. 
TABLEAU 3 (suite)

Cas d'ectromélie bithoracique à allure héréditaire relevés dans la littérature

\begin{tabular}{|c|c|c|c|}
\hline $\begin{array}{c}\text { Cas } \\
\mathbf{n}^{0}\end{array}$ & $\begin{array}{l}\text { Nom des observateurs } \\
\text { et expérimentateurs, } \\
\text { date et lieu }\end{array}$ & $\begin{array}{l}\text { Renseignements descriptifs } \\
\text { et génétiques }\end{array}$ & Auteurs \\
\hline 7 & $\begin{array}{l}\text { Les auteurs, à l'École vété- } \\
\text { rinaire de Lyon. }\end{array}$ & $\begin{array}{l}\text { Une chienne ectromèle }\left({ }^{1}\right) \text { a mis bas, en } \\
2 \text { portées, } 5 \text { petits normalement con- } \\
\text { formés. }\end{array}$ & $\begin{array}{l}\text { LESBRE et PEChEROT } \\
\quad(\mathrm{I} 9 \mathrm{I} \mathrm{I})\end{array}$ \\
\hline 8 & $\begin{array}{l}\text { L'auteur, à l'École vétéri- } \\
\text { naire d'Alfort, décrit un } \\
\text { animal fourni en I9I3 } \\
\text { par M. HoLLARD, vétéri- } \\
\text { naire a Guignes-Rabutin } \\
\text { (Seine-et-Oise). }\end{array}$ & $\begin{array}{l}\text { Une chienne ectromèle bithoracique se } \\
\text { déplaçait rapidement et avec facilité } \\
\text { sur les membres pelviens, en démarche } \\
\text { plantigrade. Pendant la marche, les } \\
\text { scapulae effectuaient des mouvements } \\
\text { parfaitement visibles qui répondaient } \\
\text { à ceux qu'auraient accompli des mem- } \\
\text { bres normaux. }\end{array}$ & DECHAMBRE (I9I4). \\
\hline 9 & $\begin{array}{l}\text { Sujet originaire de Kragu-- } \\
\text { jevac (Yougoslavie) et re- } \\
\text { cueilli à la Faculté vété- } \\
\text { rinaire de Zagreb. }\end{array}$ & $\begin{array}{l}\text { Un chien mâle ectromèle de race fox- } \\
\text { terrier a vécu I } 2 \text { ans; ill se déplaçait } \\
\text { sur ses membres pelviens. }\end{array}$ & Anonyme (1936). \\
\hline
\end{tabular}

(1) Accouplée sans doute à des mâles normaux.

Ainsi, les observations du passé apparaissent fort semblables entre elles et rappellent les nôtres tant sur le plan descriptif que génétique. Cependant, de là à proposer l'hypothèse selon laquelle il s'agirait d'une seule et même mutation dans tous les cas, il y a loin. Il, faut, en effet, tenir compte de la tératogénèse accidentelle qui peut éclairer certaines formes d'ectromélie et du phénomène de mimique qui expliquerait plus facilement peut-être que la migration, l'apparition en des lieux si éloignés et à des époques si diverses d'anomalies phénotypiquement semblables.

Reçu pour publication en août $\mathrm{I} 968$.

\section{SUMMARY}

\section{HEREDITARY BITHORACIC FCTROMELIA IN DOGS}

Eighteen male and female dogs of the same family were observed to have a bithoracic ectromelia.

The skeleton of the forelimbs has only a scapula; Yama, the mother, presented a unique case i.e. on each side of her trunk there was a stump of the humerus adjoining the scapula. hare-lip.

The observed anomaly is simple : but, in one of the puppies this was accompanied by a 
Ectromelian animals appear able to survive and produce offspring but the puppies are handicapped by the infirmity which prevents them from suckling properly. Hence the high postnatal mortality rate.

The phenotypical ratios observed in $\mathrm{F}_{1}, \mathrm{~F}_{2}$, and back-crosses, indicate a monofactorial, autosomal, recessive determinism.

Our observations agree with many points already published. However, the incomplete and dispersive nature of previous findings prevents us from stating categorically that this condition is one and the same mutation.

\section{REFÉRENCES BIBLIOGRAPHIQUES}

ANonyme, 1936. Amelia anterior bei einem Foxterrier. Tierärztl. Rundsch., 42, 675.

BARRIER, I883, in CAGNY. Un cas d'ectromélie sur le cheval, hérédité des anomalies sur les animaux. Discussion. Bull. Soc. centr. méd. vét. Paris, N.S., 1, I89-19o.

BLIN, P. C. 1958. Vergleichend-anatomische Untersuchugen über das Ganglion stellatum der Haussäugetiere sowie über seine Morphogenese an Hand von makroskopischen Befunden. Verh. Anat. Ges., 55. Versamml. Frankfurt /Main, 74-89.

Blin P. C., Ovejero del Agua F., 1952. Ectromélie double et totale chez la Chèvre. Rec. Méd. vét., 128, 374-38r.

BURNS M., Fraser M. N., 1966. Genetics of the dog. 2nd ed. Oliver and Boyd, London, 230 pp.

Dechambre P., I914. Traité de zootechnie. I. Zootechnie générale. Ch. Amat, Paris, 82-83.

GEOFFROY ST HILARRE I., I 836. Histoire générale et particulière des anomalies de l'organisation chez l'homme et les animaux, 3, 206-237. J. B. Baillière, Paris.

HEDIGER H., SIRCOM G., r955. Studies of the psychology and behaviour of captive animals in zoo and circuses. Butterworths scientific publications, London.

LADRAT J., 1967. Ectromélie bithoracique chez le chien. Carnivore genet. Newslet. (2), 29.

L,ESBRE F. X., FORGEOT E., 1902. Étude anatomique de 5 animaux ectromèles suivie de considérations générales sur l'ectromélie. J. Anat. Physio., 38, r78-192.

I ESBRe F. X., Pecherot R., IgIx. Étude de quelques monstres appartenant à un nouveau genre dit schistomele de la famille des ectroméliens. Bull. Soc. Sci. Vét. Lyon, 14, 52-79.

LISI, r909. Monstruosité (ectromélie) observée héréditairement chez quelques petits chiens. Rev. vét., 34, 432 .

Miler M. E., Christensen G. C., Evans H. E., I964. Anatomy of the dog. W. B. Saunders company, Philadelphia and London, 94I pp.

Salmon J., 1908. Sur le système nerveux des ectroméliens. C. R. Soc. Biol., 74, 13I-133.

SPENGLER H., 195o. "Brachiale Peromelie der Katze unter besonderer Berücksichtigung der neurologischen Verhältnisse ". Vet.-med. Diss. Zürich, 53 pp.

Verger J. M., 1967. Anomalies et monstruosités dans l'espèce canine. Thèse Doct. vét. Alfort, $145 p p$. 